\title{
Les leçons et les enjeux du mouvement coopératif agraire à
} Cuba

\section{Lessons and issues for the agrarian cooperative movement in}

Cuba

\section{Lecciones y desafíos del movimiento cooperativo en Cuba}

Jacques Marzin

Numéro 328, avril 2013

Les coopératives agricoles des « Sud » : quels enseignements pour les " Nord"?

Agricultural Cooperatives in the "South »: What Lessons for the « North »?

URI : https://id.erudit.org/iderudit/1015528ar

DOI : https://doi.org/10.7202/1015528ar

Aller au sommaire du numéro

\section{Éditeur(s)}

Association Recma

\section{ISSN}

1626-1682 (imprimé)

2261-2599 (numérique)

Découvrir la revue

\section{Citer cet article}

Marzin, J. (2013). Les leçons et les enjeux du mouvement coopératif agraire à Cuba. Revue internationale de l'économie sociale, (328), 63-74.

https://doi.org/10.7202/1015528ar

\section{Résumé de l'article}

Prochainement, le mouvement coopératif agricole à Cuba s'ouvrira à de nouvelles fonctions de service, d'approvisionnement et de commercialisation. Cependant, il ne concerne jusqu'à présent que la production. De multiples formes coopératives y diffèrent par le niveau et les types de mise en commun des moyens de production (terres, mécanisation, commercialisation). L'expérience de cinquante ans de ces coopératives de production (et donc leur évolution sur plusieurs générations) est riche d'enseignements dans une activité comme l'agriculture, où les investissements nécessitent des temps d'amortissement longs et posent des problèmes de transmission intergénérationnelle particuliers. Ceux-ci portent particulièrement sur la performance du secteur privé coopératif, sur l'inventivité institutionnelle des formes de tenure du foncier et de propriété du capital productif et sur les difficultés de transmission des formes de production privée collective. 


\title{
LES LEÇONS ET LES ENJEUX DU MOUVEMENT COOPÉRATIF AGRAIRE À CUBA
}

par Jacques Marzin*

\begin{abstract}
* UMR ART-Dév, Centre de coopération internationale en recherche agronomique pour le développement, Montpellier. Mél. : jacques. marzin@cirad.fr.
\end{abstract}

Prochainement, le mouvement coopératif agricole à Cuba s'ouvrira à de nouvelles fonctions de service, d'approvisionnement et de commercialisation. Cependant, il ne concerne jusqu'à présent que la production. De multiples formes coopératives y diffèrent par le niveau et les types de mise en commun des moyens de production (terres, mécanisation, commercialisation). L'expérience de cinquante ans de ces coopératives de production (et donc leur évolution sur plusieurs générations) est riche d'enseignements dans une activité comme l'agriculture, où les investissements nécessitent des temps d'amortissement longs et posent des problèmes de transmission intergénérationnelle particuliers. Ceux-ci portent particulièrement sur la performance du secteur privé coopératif, sur l'inventivité institutionnelle des formes de tenure du foncier et de propriété du capital productifet sur les difficultés de transmission des formes de production privée collective.

\section{Lessons and issues for the agrarian cooperative movement in Cuba}

The agricultural cooperative movement in Cuba is about to take on new functions in services, procurement and marketing. Up to now, it has only dealt with production. There are multiple forms of cooperatives that differ by the level and types of ownership of the means of production (land, machinery, and marketing). The fifty-year experience of these producer cooperatives (and thus their evolution over several generations) provides valuable lessons for a business such as farming, where investments have a long payback period and pose specific problems for intergenerational transmission. They relate particularly to the performance of private sector cooperatives, institutional creativity in terms of the forms of land tenure and ownership of physical capital, and the difficulties of passing on private collective forms of production.

\section{Lecciones y desafíos del movimiento cooperativo en Cuba}

Próximamente, el movimiento cooperativo agrario en Cuba se abrirá a nuevas funciones de servicio, de compras y de comercialización. Sin embargo, esto solo concierne hasta hoy la producción. Incluye múltiples formas cooperativas que se diferencian por el nivel y los tipos de las puestas en común de los medios de producción (tierras, mecanización, comercialización). La experiencia de cincuenta años de estas cooperativas de producción (y pues de su evolución sobre varias generaciones) es rica de enseñanza en una actividad como la agricultura, donde las inversiones necesitan largos tiempos de amortización y suponen la capacidad de transmisión entre generaciones. Estas lecciones conciernen particularmente la eficiencia productiva del sector privado cooperativo, las innovaciones institucionales al respecto de la tenencia de la tierra y de la propiedad del capital productivo, y las dificultades de transmisión de las formas de producción privada y colectiva. 


\section{Introduction}

La république de Cuba importe environ $70 \%$ de sa consommation alimentaire, pour un coût en devises de l'ordre de 1,5 milliard d'euros. C'est la raison pour laquelle elle s'est engagée dans de profondes transformations de son agriculture visant la substitution des importations alimentaires: arrivée de nouveaux actifs, extension du domaine marchand, modification réglementaire concernant les coopératives, prochaines ouvertures de magasins d'intrants... Le secteur privé, largement organisé sous forme de coopératives de production, joue un rôle clé dans la réussite de ces évolutions: avec $24 \%$ de la surface agricole utile (SAU), il produit $57 \%$ de l'alimentation (Nova, 2011).

Cet article présente la première partie d'un travail de recherche qui vise à analyser, à dix ans d'intervalle, le développement de vingtcinq coopératives agricoles cubaines. Il a mobilisé une analyse bibliographique des dynamiques agraires et des systèmes coopératifs, mais aussi l'étude des principaux textes réglementaires régissant le fonctionnement de l'agriculture non étatique cubaine. Les conséquences institutionnelles de ces évolutions ont été mises en perspective par la connaissance des réalités agraires cubaines qu'a acquise l'auteur grâce à un partenariat de plus de dix ans avec des organisations cubaines ${ }^{(1)}$. L'article synthétise le cadre historique de l'agriculture cubaine et de ses coopératives. Il en explicite la configuration complexe et pointe ses spécificités. Il discute ensuite les enjeux de ce secteur et les leçons qui peuvent en être tirées.

(1) Que soient ici particulièrement remerciés Juan José León, ancien vice-président de l'Anap et ancien directeur des relations internationales du ministère cubain del'Agriculture, et Teodoro López Betancourt, ancien responsable du système de vulgarisation agricole, qui ont bien voulu répondre à mes questions et me faire partager leur extraordinaire convivencia avecles agriculteurs cubains.

\section{De l'héritage colonial à l'agriculture familiale d'aujourd'hui}

A Cuba, l'agriculture familiale a longtemps été marginale au regard des autres formes de production de grande échelle (latifundia, agro-business, fermes d'Etat).

\section{Un héritage colonial}

La ruralité s'y est construite autour de villas $^{(2)}$. A l'exception de trois zones d'agriculture paysanne (Pinar del Río, Holguín et la ceinture maraîchère de La Havane) marquées par un habitat dispersé, le territoire s'est organisé près de centres urbains et de centrales sucrières ou de bâtiments des latifundios où se concentraient les travailleurs (la plupart, esclaves, dans les bateys ${ }^{(3)}$ ) et les colons

(2) Pôles urbains autour desquels l'île s'est structurée, liés à l'histoire coloniale.

(3) Baraquements où logeaient les esclaves, àl'écart des autres habitations. les plus pauvres. Cette organisation spatiale s'est peu transformée au cours du XIX siècle, malgré l'arrivée d'un colonat d'origine européenne dans les zones de production sucrière visant à éviter que la faible population de l'île (1 million en 1841, soit une densité de 9 habitants par kilomètre carré; 2 millions en 1907) ne devienne à majorité d'origine africaine. A cette même date, le secteur primaire (agriculture, pêche, mines) ne dépassait pas $48 \%$ de la population active (recensement de 1907). La surface mise en valeur par l'agriculture 


\section{Figure 1}

\section{Evolution des formes de tenure à Cuba, 1931-2010}

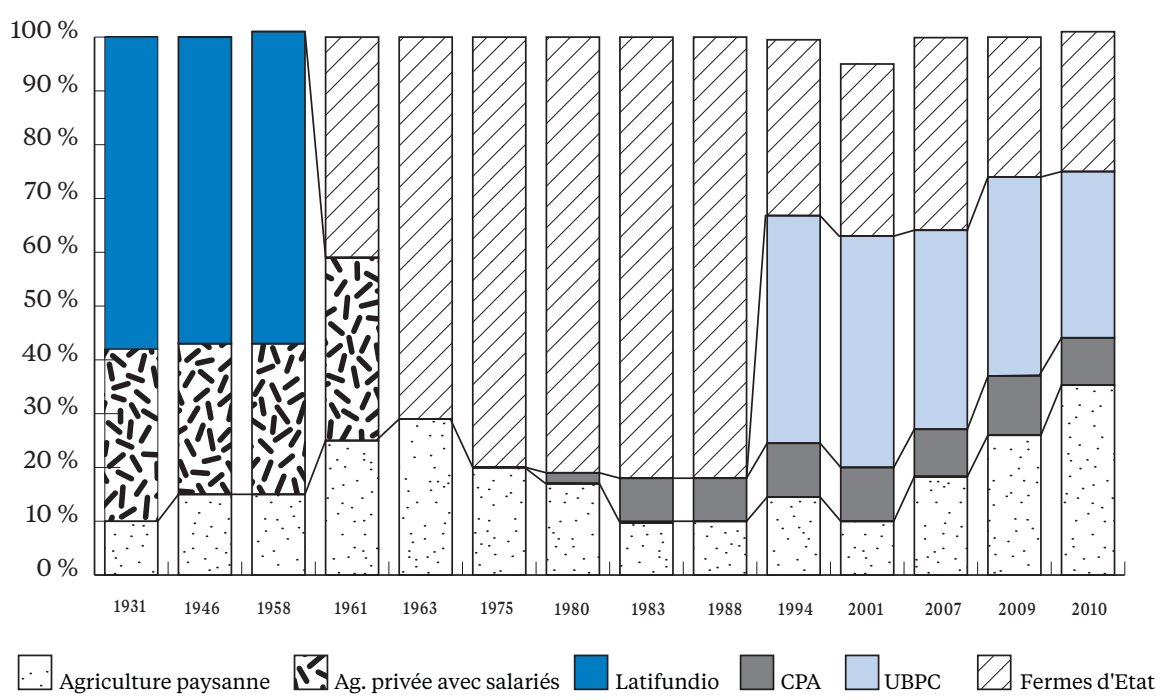

Sources: calculs de l'auteur sur la base des données issues de Carlos Rafael Rodriguez (1965), de Deere et al. (1994), de Douzant-Rosenfeld et al. (1995), de Valdès Paz (1997), de ONE (2007), d’Arias Guevara (2009), de Piñeiro (2011).

paysanne a longtemps été inférieure à $20 \%$ de la surface agricole utile. Telle est la situation au début du $\mathrm{XX}^{\mathrm{e}}$ siècle, au cours duquel des évolutions plus fortes vont se dérouler (figure 1).

\section{Une agriculture duale}

L'agriculture cubaine s'est développée autour de produits d'exportation (sucre, agrumes et tabac) et de produits vivriers tropicaux. Les deux premiers produits sont issus d'une agriculture capitalistique. Les capitaux américains y remplacent progressivement ceux de l'ancienne puissance coloniale espagnole. Les autres produits sont plus largement cultivés par l'agriculture familiale. Cette agriculture duale se maintiendra après la révolution de 1959, sous des formes renouvelées, l'agriculture capitalistique avec des salariés étant remplacée par des fermes d'Etat.

Après l'avènement de la révolution socialiste, les deux réformes agraires ont permis à des métayers, des fermiers ou des salariés agricoles d'accéder à la propriété (Alvarez, 2006). Pourtant, une partie d'entre eux ont préféré devenir salariés des nouvelles fermes d'Etat. C'est le premier pic de l'agriculture paysanne. L'articulation de la réforme agraire avec des réformes sociales de profondeur (alphabétisation, remembrement, concentration de l'habitat rural dans des villages, scolarisation, bourses d'études, développement de services publics dans des pôles urbains multiples) a paradoxalement favorisé la mobilité sociale des enfants de paysans, bénéficiaires de la réforme agraire, vers l'administration publique d'encadrement de la production agricole et, plus généralement, vers les autres secteurs productifs (Jiménez, Almaguer, 2003). La transmission de la terre en propriété ne peut 
se faire que par héritage et avec la condition d'une mise en valeur directe, aucun marché foncier n'étant toléré. Jusqu'en 1990, l'agriculture paysanne semblait donc vouée à s'éteindre progressivement.

Cette tendance s'est cependant infléchie avec l'effondrement du bloc socialiste européen, qui a mis fin à la division internationale des productions du camp socialiste et donc à la spécialisation cubaine dans le sucre (Valdés, 1997). L’agriculture paysanne, qui avait maintenu des pratiques agricoles utilisant peu d'intrants, a été fondamentale pour la redynamisation d'une production agricole diversifiée (Rosset et al., 2011), alors que l'agriculture d'Etat, fruit du modèle intensif de la Révolution verte, s'effondrait. Les réformes de 1993 visant à une décentralisation (notamment dans la commercialisation et la politique de rémunération) et à une désétatisation de l'agriculture (transformation de fermes d'Etat en coopératives, les UBPC) l'ont aussi renforcée (Deere et al., 1994). Elle a alors attiré de nouveaux actifs. Cela explique la complexité des formes de production cubaine.

\section{L'agriculture familiale d'aujourd'hui}

La crise alimentaire de 2008, jointe au ralentissement économique qui fragilise les exportations cubaines de nickel, renforce la nécessité d'une stratégie de substitution aux exportations. Si le secteur privé, avec $20 \%$ des terres, produisait $80 \%$ de l'alimentation consommée sur l'île, de nombreuses terres restaient improductives (essentiellement dans les UBPC et les fermes d'Etat). La dernière redistribution foncière, marquée par une attribution en usufruit de terres considérées comme inexploitées, y répond en partie. Elle consiste en une croissance extensive de la production agricole par la redistribution sous forme d'usufruit de terres arables non exploitées. Au 31 octobre 2012 (Delgado Guerra, 2012), 172000 personnes et 2200 entités juridiques ont été attributaires de 8,75 ha en moyenne, soit une surface totale de 1523000 ha, qui correspond à $15 \%$ de la SAU cubaine. A l'échelle de la France, cela rassemblerait les surfaces des régions Aquitaine et Midi-Pyrénées.

Cela explique que l'agriculture familiale dépasse aujourd'hui son niveau historique de mise en valeur de plus de $20 \%$ de la SAU. C'est le second pic de l'agriculture paysanne. Bien qu'aucune publication ne puisse à ce jour affiner les portraits types de ces attributaires, le ministère de l'Agriculture estime que la moitié d'entre eux sont des paysans qui agrandissent leurs exploitations, l'autre moitié étant des néo-agriculteurs. La plupart de ces derniers renforcent la dynamique de l'agriculture urbaine et périurbaine. On peut donc dire que l'agriculture familiale cubaine n'est plus exactement une agriculture paysanne ni totalement rurale.

\section{Un coopératisme agraire multiforme}

Les coopératives avant la révolution cubaine ne concernaient pas spécifiquement le monde agricole. Il existait des coopératives de transport et de services, et le monde agricole était plutôt structuré sous forme d'associations (Piñeiro, 2012). La révolution cubaine a promu de profondes réformes agraires, initiant le développement du mouvement coopératif agraire, qui ne connaîtra cependant sa première loi qu'en 1982. 


\section{Tableau 1}

Les principales formes actuelles de tenures à Cuba

\begin{tabular}{|c|c|c|c|c|c|c|}
\hline & 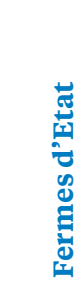 & 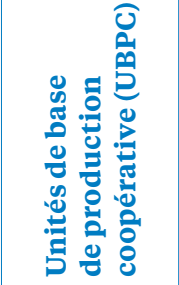 & 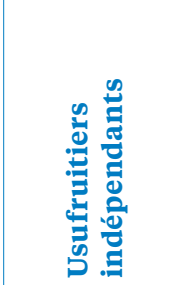 & 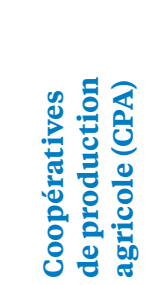 & 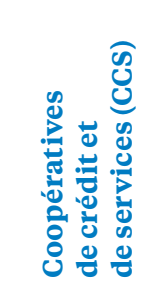 & 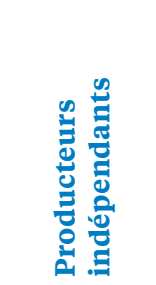 \\
\hline Sol & Etat & $\begin{array}{l}\text { Etat: usufruit } \\
\text { gratuit }\end{array}$ & $\begin{array}{l}\text { Etat: } \\
\text { usufruit } \\
\text { gratuit }\end{array}$ & $\begin{array}{l}\text { Collective: } \\
\text { coopérateurs }\end{array}$ & $\begin{array}{l}\text { Individuelle: } \\
\text { coopérateurs } \\
\text { Usufruitiers }\end{array}$ & $\begin{array}{l}\text { Individuelle: } \\
\text { producteurs }\end{array}$ \\
\hline $\begin{array}{l}\text { Moyens } \\
\text { de production }\end{array}$ & Etat & $\begin{array}{l}\text { Collective: } \\
\text { coopérateurs }\end{array}$ & $\begin{array}{l}\text { Individuelle: } \\
\text { producteurs }\end{array}$ & $\begin{array}{l}\text { Collective: } \\
\text { coopérateurs }\end{array}$ & $\begin{array}{l}\text { Collective: } \\
\text { coopérateurs }\end{array}$ & $\begin{array}{l}\text { Individuelle: } \\
\text { producteurs }\end{array}$ \\
\hline Etatique & & & Coopératif & & & Individuel \\
\hline
\end{tabular}

Source: auteur.

\section{L'émergence de nouvelles formes de coopérative}

Trois grands types de coopérative de production structurent le monde rural cubain (tableau 1). Les coopératives de crédit et de services (CCS) ont été créées rapidement après la seconde réforme agraire (1963), pour faciliter l'organisation des bénéficiaires de cette dernière. Les paysans sont propriétaires de leurs terres, cultivent leur ferme de manière indépendante, mais se regroupent pour l'achat de matériel et la commercialisation d'une partie de leurs produits (Rivera et al., 2006). Depuis les années 2000, certaines d'entre elles sont dites fortalecidas ${ }^{(4)}$ si elles ont, entre autres, recruté un comptable et un administrateur pour leur gestion.

Les coopératives de production agricoles (CPA) ont été créées après

(4) « Renforcées », notamment pour la commercialisation. le premier congrès du Parti communiste cubain (PCC), en 1976. Les paysans privés y apportent leurs terres, qui deviennent une propriété privée collective, de même que les autres moyens de production. L'organisation du travail est elle aussi collective. Après une phase de centralisation, les CPA évoluent, avec la création de sous-unités où des collectifs réduits de coopérateurs ont plus d'autonomie. Elles peuvent dépasser le millier d'hectares.

Les unités de base de production collective (UBPC) ont, elles, été créées après 1993. Elles répondaient à la nécessité de décentraliser les fermes d'Etat (Pérez et al., 2006). La terre appartient à l'Etat, elle est donnée en usufruit à cette structure juridique. Les autres moyens de production appartiennent au collectif. Comme les CPA, une décentralisation progressive tend à recréer en leur sein de plus petites unités ayant davantage d'autonomie (Nova, 2004). Durant les années 1978 et 1979, onze unions municipales de coopératives agraires (UMCA) ont été testées. Ces regroupements de coopératives (CCS 
et CPA) ont eu pour mission de développer des fonctions d'appui (approvisionnement, assistance technique, commercialisation). L'expérience a été écourtée, car elle provoquait des recoupements avec les fonctions des entreprises d'Etat chargées des mêmes fonctions.

Elles ont été les seules expériences de coopératives de second degré depuis le début de la révolution. Aucune synthèse ou publication ne permet d'en tirer les leçons.

\section{Les spécificités du coopératisme cubain}

Le développement d'un système coopératif dans une économie socialiste induit des spécificités qui le distinguent de ceux existant dans les économies de marché. Tout d'abord, tant que la nouvelle loi sur les coopératives n'est pas publiée, il s'agit uniquement de coopératives de production. Elles gardent une taille relativement réduite (de quelques coopérateurs à une grosse centaine, rarement plus). Celle-ci se prête donc à un fonctionnement démocratique direct, sans structure intermédiaire. L'unité de lieu facilite aussi les contacts entre élus, dirigeants et coopérateurs.

Ensuite, les coopératives s'insèrent dans un système économique encore essentiellement marqué par une coordination hiérarchique. Il n’existe pas pour l'instant de marché des intrants, ni des biens d'équipement: ils sont alloués par une entreprise spécialisée du ministère de l’Agriculture, au niveau des communes. La commercialisation reste en partie gérée par des entreprises d'Etat, et les formes privées de commercialisation $\left(\right.$ agromercados $^{(5)}$, par exemple) nécessitent des conventions avec les pouvoirs politiques municipaux.

Enfin, ces coopératives de production diffèrent sensiblement par la forme de propriété du foncier (individuelle, collective ou étatique). Or il n'existe pas de marché du foncier agricole: les fermes privées peuvent être transmises au sein de la famille du producteur, mais l'Etat est le seul acheteur possible si la famille ne souhaite pas garder le bien en l'exploitant. Cette dimension joue un rôle particulier, dans la mesure où il n'existe à ce jour pas de valeur patrimoniale ni de perspective de plus-value sur le foncier, donc pas de spéculation sur la valorisation par un changement d'utilisation du sol (urbanisation), ni de complication dans les successions, puisque le foncier n'ayant pas de valeur marchande il n’occasionne pas la nécessité de soulte entre héritiers.

\section{Les réformes récentes et en cours}

En juillet 2008, le décret-loi 259 définit les modalités d'un vaste processus de redistributions de terres de l'Etat considérées comme improductives (ociosas, non cultivées). Elles concernent aussi bien des personnes physiques que des personnes morales. Le décret-loi limitait la surface des terres allouées à 40 ha. Le nouveau décret-loi $n^{\circ} 300$, applicable le 9 décembre, augmente cette limite à 67 ha. L'évolution de la taille des terres mises à disposition suppose soit le développement du salariat agricole, soit celui de la mécanisation, une famille ne pouvant mettre en valeur manuellement ou avec de la traction animale presque 70 hectares. Les attributions pour les surfaces les plus petites concernent des terres dans les zones urbaines ou en périphérie. Les nouveaux attributaires peuvent soit rester indépendants, soit intégrer 
ou constituer des CCS. Avec le nouveau décret-loi n 300, ils devront intégrer obligatoirement une structure coopérative, mais ils pourront choisir entre les différentes formes existantes (CCS, CPA ou UBPC) et définir leur forme de participation dans les CPA ou UBPC (travail individuel ou collectif). Cela changera la nature de ces coopératives, car elles intégreront des personnes ayant et des trajectoires personnelles et des droits fonciers différents. L'autorisation donnée aux usufruitiers de construire une maison et des bâtiments agricoles sur le terrain octroyé est une nouveauté importante. Ces constructions sont transmissibles aux héritiers, pour peu qu'ils continuent à y vivre et à y travailler. Cette mesure pourra peut-être faciliter la stabilité des nouveaux agriculteurs (Linton Page, 2010).

\section{L'ÉVOLUTION DES UBPC}

En août 2012, une résolution (574/12) actualisait les règles de fonctionnement des coopératives de type UBPC (Gonzalez, 2012). Ces dernières obtiennent une personnalité juridique pleine et entière, alors qu'elles dépendaient auparavant du ministère de l'Agriculture pour certaines actions ou opérations, notamment comptes en pesos convertibles. Elles pourront avoir accès directement au crédit bancaire. Les relations commerciales avec le ministère de l'Agriculture relèvent dorénavant de contrats précis (pouvant aller à plus d'une centaine de pages), et non plus de conventions non contraignantes. Elles concernent aussi bien les intrants qu'une partie de la commercialisation. L'élection des représentants des UBPC est dorénavant totalement autonome du ministère de l'Agriculture. Les mécanismes du fonctionnement démocratique de ces coopératives sont précisés, ainsi que le principe d'une démarche générale de déconcentration en collectifs de taille plus réduite.

\section{LA CRÉATION DES COOPÉRATIVES DE SECOND DEGRÉ}

La réactualisation des textes légaux sur les coopératives est en cours. Les orientations données par le VI e congrès du PCC en dessinent toutefois les grandes lignes. L'introduction de la notion de coopérative de second degré (art. 29) permettra à des coopératives de production de mutualiser des fonctions qu'elles ne peuvent à ce jour mettre en place seules (commercialisation, regroupement d'achats, mécanisation partagée, transformation des matières premières, conseil technique, gestion...) [Marzin, 2012]. De la nature des fonctions qui seront finalement autorisées dépendra l'ampleur de la mue du mouvement coopératif. Les négociations en cours au sein des autorités compétentes sont ardues, car elles mettent parallèlement en jeu une recomposition plus ou moins forte du rôle du ministère de l'Agriculture.

\section{Les enjeux des dynamiques agricoles et le rôle du mouvement coopératif}

Il est reconnu, depuis l'effondrement du bloc soviétique, que le secteur privé individuel de l'agriculture (producteurs indépendants et CCS) a une efficacité supérieure aux autres formes de production. Nova (2011b) reporte qu'avec $24,4 \%$ de la SAU les producteurs privés produisent $57 \%$ de l'alimentation du pays. Cette importance du secteur privé agricole est reconnue depuis 
le début des années 90. Les formes coopératives à propriété collective connaissent des succès variables, en général liés à la dotation en facteurs de production (ressources naturelles, mais surtout investissements subventionnés par l'Etat pour les systèmes d'irrigation, de mécanisation ou le matériel pour les cultures sous abri). La productivité du travail y est plus faible et par conséquent les niveaux de rémunération aussi, mais les conditions de vie et de travail également, ce qui explique le turn-over très élevé des «coopérateurs ", qui se considèrent souvent plus comme des salariés que comme des membres de la coopérative. Ce turn-over dépasse dans bien des cas les $30 \%$. Le très grand dynamisme du marché du travail cubain contribue à expliquer ce chiffre élevé.

\section{Faible mécanisation et bonne rémunération du travail}

Faute d'investissements notables depuis plus de vingt ans, l'agriculture cubaine est peu mécanisée. La flotte de tracteurs encore en fonctionnement est principalement utilisée pour le transport des travailleurs (qui ne logent pas, pour la majorité, sur leur lieu de travail, mais dans les villages environnants). La traction animale est largement développée pour le travail du sol (bœufs) et le transport (chevaux). De nombreuses façons culturales restent manuelles. Cela explique la très faible productivité du travail dans l'agriculture cubaine. Des chiffres issus du suivi de coopératives au début des années 2000 permettent d'estimer des ordres de grandeur. En l'absence d'investissements bouleversant les modes de production, ils restent globalement valables. Dans les unités de production laitière (secteur des coopératives), la productivité varie autour de 3000 à 7000 litres de lait par travailleur et par an et le chiffre d'affaires annuel par travailleur est de l'ordre de 200 à 300 euros. Cela correspond à une dizaine de salaires mensuels, ce qui explique que les salaires des producteurs, quoique très variables, soient dans la frange supérieure des revenus cubains (deux à trois fois le revenu moyen ${ }^{(6)}$, parfois beaucoup plus). Sans doute faut-il y voir la raison du succès de la redistribution des terres. Cependant, cette

(6) 455 pesos, soit environ 15 euros. dynamique est très fragile. D’abord, les prix agricoles aujourd'hui élevés peuvent baisser, soit par l'intervention de l'Etat (fixation de prix maximum), soit par le jeu de l'offre croissante, qui pèsera sur les cours. Les marges de progrès sont énormes, dès que des intrants sont accessibles. Ensuite, les prix des produits agricoles n'intègrent pas encore le coût réel des intrants et des équipements, actuellement largement subventionnés à cause du système de double monnaie (monnaies nationale et convertible). Ce système en transition entre une coordination hiérarchique et l'introduction progressive de mécanismes de l'économie de marché dans une situation générale de pénurie explique que le système coopératif pourra jouer un rôle essentiel dans l'évolution de l'agriculture cubaine.

\section{Propriété privée, individuelle ou collective?}

Les besoins d'investissements sont énormes: pour la mécanisation et la motorisation, mais aussi pour les infrastructures de production, d'élevage, de stockage et de transformation des produits agricoles. Ces investissements sont essentiels afin d'augmenter la productivité du travail, qui à son tour 
garantira une hausse de la rémunération. Si l'accès individuel au crédit a été élargi, la faible disponibilité d'intrants et de biens d'équipement justifie que des investissements collectifs soient opérés. Le système coopératif, de premier ou de second degré, pourra y jouer un rôle fondamental.

S’il est clair que la production étatique est amenée à se réduire à Cuba dans les prochaines années, un enjeu fort concerne la forme de propriété privée (Nova, 2004): individuelle (type coopérative de crédit et de services [CCS]) ou collective (type coopérative de production agricole [CPA]). Les CCS ont montré leur supériorité productive dans un environnement spécifique: absence de marché des intrants, pénurie de biens d'équipement. Le facteur travail y est mieux rémunéré que dans les autres formes de production. Cependant, le desserrement des contraintes à l'investissement pourrait profiter aux formes de propriété collective, d'une part pour la valorisation des biens d'équipement (économies d'échelle classiques), d'autre part dans l'organisation du travail, les avantages sociaux (Mesa-Lago, Vidal-Alejandro, 2011) et la qualité de vie. Il est clair que la teneur de la future réglementation régissant les coopératives jouera un rôle fondamental dans les évolutions de ces deux formes distinctes du coopératisme cubain (Piñeiro, 2011): les modes d'imposition (et les niveaux d'exonération fiscale), les différentiels de taxation entre un auto-emploi et un emploi salarié pèseront sur ces évolutions.

\section{Ouverture de la loi aux coopératives de services}

Des gisements énormes de productivité existent pour l'agriculture cubaine (Douzant-Rozenfield, 2002): la politique de substitution aux importations de produits alimentaires ${ }^{(7)}$ devrait se traduire par une accessibilité accrue aux intrants agricoles dans des magasins dédiés. D’importantes pertes post-récolte pourraient être réduites si une articulation plus forte existait entre la production agricole et la transformation (artisanale

(7) Les devises investies dans l'achat en devises d'intrants et de biens d'équipement viendraient réduire les dépenses en produits alimentaires. ou industrielle). Le système de commercialisation pourrait être amélioré par une efficacité renforcée du système de transport des marchandises. Ces fonctions (approvisionnement, transformation, transport et, dans une moindre mesure, commercialisation) sont encore sous la responsabilité de l'Etat. Les discussions en cours sur la loi des coopératives sont ouvertes: quels seront les domaines dans lesquels les coopératives de second degré pourront s’investir? Quelles seront les règles juridiques régissant leur exercice?

Par ailleurs, des fonctions de services d'assistance technique, de conseil juridique ou de comptabilité-gestion devraient être ouvertes aux coopératives, au-delà du secteur agricole. Là aussi, la coopération agricole pourrait se développer fortement.

\section{Conclusion: quelles transmissions intergénérationnelles?}

L'expérience de trente ans des coopératives de production agricoles à Cuba permet de pointer deux éléments. Le choix qui a été fait dans les années 70 de la mise en commun de propriétés individuelles et de leur fusion dans un tout collectif sans rémunération individuelle du capital apporté (pas de 
rémunération de parts sociales ni de remboursement du capital social en cas de départ) a mis sur un pied d'égalité les coopérateurs qui apportaient des moyens de production et ceux qui entraient avec leur seule force de travail. Cette égalité n’a pas posé de problème majeur à la première génération de coopérateurs, qui trouvaient leur motivation dans l'intérêt du travail collectif, de la mécanisation et de l'amélioration de la qualité de vie (notamment grâce à la promesse de l'octroi de pensions de retraite au taux maximal aux exploitants âgés n'ayant pas forcément cotisé dans leur jeunesse). En revanche, ce choix s'est rapidement traduit par un sentiment de « désappropriation » du bien collectif. Les coopérateurs sont devenus plus des salariés que des membres de la coopérative. C'est très net pour la seconde génération d'entre eux, qui ont souvent choisi un emploi hors de la coopérative et n'ont gardé que leur logement au sein de celle-ci.

L’absence de valorisation patrimoniale par un marché foncier a donné une grande souplesse aux trajectoires des coopérateurs. Si certains ont été agriculteurs toute leur vie, nombreux sont les actifs dans l'agriculture cubaine qui ont eu des expériences professionnelles au sein d'autres secteurs de l'économie ou dans d'autres fonctions que la production agricole. Ces carrières riches militent pour une certaine déconnexion entre la propriété du capital productif (au moins le foncier) et le patrimoine familial.

L'expérience cubaine a donc un intérêt particulier dans l'analyse de formes d'organisation coopérative dans lesquelles le droit d'usage sur tout ou partie des moyens de production, notamment le foncier, joue un rôle fondamental. La manière dont le secteur coopératif saura réagir aux nouvelles donnes de la politique économique cubaine sera porteuse d'enseignement pour l'ensemble du secteur de l'économie sociale et solidaire. 


\section{BiBLIOGRAPHIE}

Alvarez Licea M. D., 2006,

«Estructuras de producción y sostenibilidad en la agricultura campesina », in Fúnez F. y Pérez N. (eds), Transformando el campo cubano, Actaf, La Habana.

Arias Guevara, MDLA, 2009, "Cuba, reforma y transformacion agraria: la crisis de los noventa y el proceso de desestatalizacion de la agricultura ", Ideas, vol. 3, n 1 , p. 6-29.

Deere C. D., Perez N., Gonzales N., 1994, "The view from below: Cuban agriculture in the special period in peacetime ", Journal of peasant studies, vol. $21, n^{\circ} 2$, p. 194-234.

Delgado Guerra S., 2012,

«Decreto-Ley 300: una inyección a la productividad ", Periódico Granma, La Habana, año 16, n²295, jueves 25.

\section{Douzant-Rozenfield D., Linck, T.} et Zequeira M., 1995,

"Se nourrir à Cuba: les enjeux de l'agriculture dans la région de La Havane », Les Cahiers des Amériques latines, $\mathrm{n}^{\circ} 19, \mathrm{p} .35-63$.

Douzant-Rozenfield D., 2002, «Les défis du développement de l'agriculture cubaine ", Les Cahiers des Amériques latines, $\mathrm{n}^{\circ} 31-32, \mathrm{p} .21$.

Gonzalez, CAM, 2012, Ensayo critico sobre el cooperativismo agricola en Cuba, Revista Tema.

\section{Jiménez Guethón R.} Almaguer Guerrero R., 2003,

«El cooperativismo cubano: historia, presente y perspectivas ", UniRcoop, vol. 1, n² 2, p. 178-200.
Linton Page T., 2010,

"Can the State create campesinos?

A comparative analysis of the Venezuelan and Cuban repeasantization programmes ", Journal of agrarian change, vol. $10, \mathrm{n}^{\circ} 2$, p. $251-272$.

Marzin J., 2012, « Réforme économique ou cure de minceur du modèle social? Les orientations du VI ${ }^{\mathrm{e}}$ congrès du Parti communiste cubain ", Les Cahiers des Amériques latines, nº 67, p. 10.

Mesa-Lago C., Vidal-Alejandro P., 2011, " The impact of the global crisis on Cuba's economy and social welfare ", Journal of Latin

American studies, vol. 42, n 4, p. 689-717.

Nova González A., 2011, « La propiedad en la economía cubana », Revista Tema.

Nova González A., 2004,

«El cooperativismo: línea de desarrollo en la agricultura cubana 1993-2003", Latin American Studies Association, $\mathrm{XXV}^{\mathrm{e}}$ international congress, Las Vegas, Nevada, USA.

Nova González A., 2011b, « Valoración del impacto de las medidas más recientes en los resultados de la agricultura ", in Perez Vilanueva OE, Political economy of change in Cuba, Bilder, p. 87-104.

ONE, 2007, Anuario estadístico 2006, Oficio nacional de estadística, La Habana, 150 p.

Pérez Rojas N., Echevarría León D., 2006,

« Las unidades básicas de producción cooperativa cubanas (1993-2003): análisis para un debate », in de Grammont HC, La construcción de la democracia en el campo latinoamericano, Clacso, Consejo latinoamericano de ciencias sociales, Buenos Aires, p. 243-259. 
Piñeiro Harnecker C. (eds), 2011, Cooperativas y socialismo: una mirada desde Cuba, Editoral Caminos, La Habana, 419 p.

Piñeiro Harnecker C., 2012,

«La contribución de las cooperativas para la actualización del modelo económico cubano ", in Nova A., Miradas de la economía cubana, CEEC, La Habana.

\section{Rivera Rodríguez C. A., Labrador Machín O.,} Alfonso Alemán J. L., 2006, «El cooperativismo agropecuario: una vía para el desarrollo económico, productivo y social en el modelo económico cubano ", Revista de ciencias sociales, vol. XII, n² 2 , Maracaibo.
Rodríguez, Carlos Rafael, 1965,

«La revolución cubana y el campesinado », Revista Cuba socialista, $\mathrm{n}^{\circ} 53$.

Rosset P. M., Sosa B. M., Jaime A. M., Lozano D. R., 2011,

« The campesino-to-campesino agroecology movement of Anap in Cuba: social process methodology

in the construction of sustainable peasant agriculture and food sovereignty ", The Journal of peasant studies, vol. 38 , issue 1 .

Valdès Paz J., 1997, Procesos agrarios en Cuba 1959-1995, Cali, Instituto cubano del libro. 